OPEN ACCESS

Edited by: Claudia Gianelli,

University of Potsdam, Germany

Reviewed by:

Lucia M. Sacheli,

Sapienza University of Rome, Italy

Lincoln J. Colling,

Australian Catholic University,

Australia

${ }^{*}$ Correspondence:

Luisa Sartori,

Dipartimento di Psicologia Generale,

Università di Padova, Via Venezia 8,

35131 Padova, Italy

luisa.sartori@unipd.it

Specialty section:

This article was submitted to

Cognition,

a section of the journal

Frontiers in Psychology

Received: 27 January 2015

Accepted: 17 April 2015

Published: 01 May 2015

Citation:

Sartori L and Betti S (2015)

Complementary actions.

Front. Psychol. 6:557.

doi: 10.3389/fpsyg.2015.00557

\section{Complementary actions}

\author{
Luisa Sartori $^{1,2 *}$ and Sonia Betti ${ }^{1}$ \\ 'Dipartimento di Psicologia Generale, Università di Padova, Padova, Italy, ${ }^{2}$ Cognitive Neuroscience Center, Università di \\ Padova, Padova, Italy
}

Complementary colors are color pairs which, when combined in the right proportions, produce white or black. Complementary actions refer here to forms of social interaction wherein individuals adapt their joint actions according to a common aim. Notably, complementary actions are incongruent actions. But being incongruent is not sufficient to be complementary (i.e., to complete the action of another person). Successful complementary interactions are founded on the abilities: (i) to simulate another person's movements, (ii) to predict another person's future action/s, (iii) to produce an appropriate incongruent response which differ, while interacting, with observed ones, and (iv) to complete the social interaction by integrating the predicted effects of one's own action with those of another person. This definition clearly alludes to the functional importance of complementary actions in the perception-action cycle and prompts us to scrutinize what is taking place behind the scenes. Preliminary data on this topic have been provided by recent cutting-edge studies utilizing different research methods. This mini-review aims to provide an up-to-date overview of the processes and the specific activations underlying complementary actions.

Keywords: action observation, perception-action coupling, social interactions, motor resonance, transcranial magnetic stimulation

\section{Introduction}

Motor resonance is defined as the subliminal activation of the motor system-and of the imitative response-while observing actions performed by others (reviewed in Heyes, 2011). Gallese (2001) explained that: "when we observe actions performed by other individuals our motor system 'resonates' along with that of the observed agent" (pp. 38-39). Numerous neurophysiological studies have in fact demonstrated that a motor resonant mechanism is at work in the motor, premotor, and the posterior parietal cortices when individuals are instructed to observe goal-directed actions being executed by another or others (for review, see Fadiga et al., 2005; Heyes, 2011; Rizzolatti et al., 2014). The discovery of mirror neurons in monkeys provided the physiological model for this perception-action coupling mechanism (Rizzolatti and Craighero, 2004). Located in the ventral premotor cortex (area F5) and the posterior parietal cortex, mirror neurons were found to fire both when a monkey carried out a goal-directed action as well as when it observed that same action being performed by another subject (di Pellegrino et al., 1992). Motor resonance appears then to pre-activate the motor system of an observer in order to represent and interpret the movements of another person even before the "go" signal has been given and activation remains for the most part on an unconscious level (Costantini et al., 2011).

While actions that are observed and those that are being planned appear functionally equivalent (Knoblich and Flach, 2001), it is unclear if the visual representation of an observed action inevitably leads to its motor representation. This is particularly true with regard to complementary (from Latin complementum; i.e., that fills up) actions, a specific class of movements which differ from -although 
interacting with- an observed action (Sebanz et al., 2006; Knoblich et al., 2011). In the case, for example, that someone hands us a mug by its handle, we will automatically, without giving it a second thought, grab the mug using a whole-hand-grasp (the most appropriate grasping posture in this particular situation). The types of grasps adopted by the two interacting agents are incongruent, but they are nevertheless appropriate and complementary.

As a working definition, complementary actions refer here to any form of social interaction wherein two (or more) individuals coordinate and mutually complete their incongruent actions, rather than performing imitative behaviors. In this respect, we can define as complementary affordances all the action possibilities in which suitable motor programs aiming to bring a joint goal to completion are activated (such as grasping and offering a coin when seeing an open hand in sign of request). Depending on its posture and context, therefore, an extended open hand could lead to a donation, to a handshake or to an infinite number of other actions (Sartori et al., 2009). Activation of a complementary affordance is an important social tool, and it suggests that the automatic, rapid decoding of social cues influences intentional behavior in our everyday interactions, maximizing the efficiency of our responses. These examples illustrate the functional importance of complementary actions in the action-perception domain (Graf et al., 2009), and they prompt us to examine the mechanisms involved in producing those responses.

\section{Behavioral Studies of Complementary Actions}

Since the direct matching between observed and performed actions is thought to occur automatically, when we observe an action which differs from our intended action we have to inhibit the tendency to imitate (Brass et al., 2005). While the mechanism leading to automatic imitation is relatively well-studied (Heyes, 2011), it is less clear how this automatic tendency is brought under control.

Evidence that task representation plays a pivotal role in shaping our actions has been provided by a series of studies (NewmanNorlund et al., 2007a,b; van Schie et al., 2008b; Poljac et al., 2009) in which participants were explicitly instructed to prepare imitative or complementary actions after viewing a virtual actor grasp a manipulandum using either a precision grip (PG; i.e., opposition between the index finger and thumb) or a wholehand grasp (WHG; i.e., opposition of the thumb with the other fingers). As expected, participants were faster at preparing their response in imitative contexts if the action to be carried out was congruent with what they had observed. When, instead, they were expected to carry out complementary actions, they responded faster when their action was dissimilar to the one they had just observed. The task representation (imitative vs. complementary) seems then to overrule long-term stimulus-response associations, influencing the way that action-perception coupling takes place. Further evidence concerning this flexible perception-action coupling was produced by a 3D motion capture study (Ocampo and Kritikos, 2010) in which reaching and grasping parameters of congruent responses were found to improve in imitative contexts, and incongruent responses were facilitated in complementary contexts. Consistent with these findings, Longo et al. (2008) demonstrated that also the level of action coding can be modified (e.g., toward coding in terms of movements) depending on task requirements. Taken together, these data challenge the idea that action observation automatically leads to imitation in the observer and suggest that, depending on the context, observed actions can prime incongruent responses.

Recently, Sacheli et al. $(2012,2013)$ showed that participants involved in face-to-face interactions can mutually adjust their movements in time and space even in the absence of instructions to either imitate or perform a complementary response. This demonstrates that priming does not strictly depend on taskconstrains, and that humans might indeed be able to actively shift from imitative to complementary actions, thanks to neurocognitive processes that still needs to be clarified.

\section{Neuroimaging Studies of Complementary Actions}

Few studies have examined the neural circuitry behind joint actions, and in particular the human mirror neuron system's (hMNS) involvement in complementary forms of social interaction. Might the hMNS provide a substrate for complementary actions? And if not, what role do other brain systems play?

In a pioneering experiment, the response of the hMNS was specifically investigated in imitative and complementary action contexts using functional magnetic resonance imaging (fMRI; Newman-Norlund et al., 2007a,b). Signals were recorded while the participants prepared to grasp a manipulandum in one of two ways - with a WHG or a PG-after they viewed an actor carrying out that action. It was found that preparation for complementary actions resulted in an increased blood-oxygen-level-dependent (BOLD) signal in the right inferior frontal gyrus (IFG) and in the bilateral inferior parietal lobule (IPL), two core components of the mirror system (Figure 1). This finding can be explained in terms of different kinds of mirror neurons: strictly congruent mirror neurons, which respond to identical actions, both observed and performed ones, and broadly congruent mirror neurons, which respond to non-identical observed and performed actions and objects linked to them (Fogassi and Gallese, 2002). It is also possible that in the complementary condition, when participants observe an action drawing attention to an object eliciting a different action, an interplay takes place between mirror and canonical neurons with the latter responding both during the time the action is being executed and also while the objects linked to those behaviors are perceived (Rizzolatti and Craighero, 2004). The need to carry out a complementary action involving a different object might then imply a combination of mirror and canonical neurons coding for different types of actions at different times of the sequence. The hypothesis that different classes of mirror neurons serve to integrate observed and executed actions during complementary kinds of social interaction is certainly an appealing one.

Newman-Norlund et al. (2007a,b, 2008) also hypothesized that a joint action could preferentially recruit right lateralized components of the mirror system since right inferior frontal activations are linked to inhibition processes (Brass et al., 2005). Planning and executing complementary actions in this 

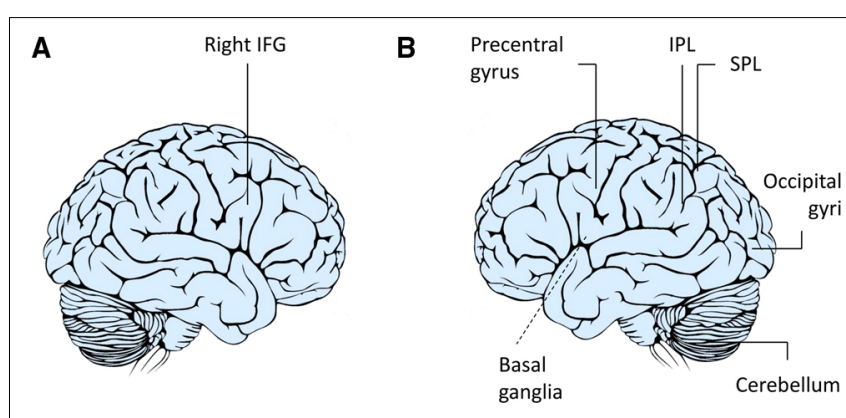

FIGURE 1 | Neuroimaging studies of complementary actions. A number of studies have suggested that the right IFG $(A)$ is not only involved when we respond to the actions of others by doing the same as they do (imitation) but also when responding with complementary actions (Newman-Norlund et al., 2007a,b, 2008; Ocampo et al., 2011; Shibata et al., 2011). In contrast, others hypothesize that the flexibility required during complementary actions requires a large network (B) including the IFG, IPL, superior parietal lobule (SPL), precentral gyrus, basal ganglia, middle and temporal occipital gyri, and cerebellum to be involved in integrating one's own actions to those of others (Kokal et al., 2009; Kokal and Keysers, 2010).

framework would mean, first of all, actively inhibiting the natural tendency to imitate observed actions. In the light of recent debates revolving around mirror mechanisms (Gallese and Sinigaglia, 2011; de Bruin and Gallagher, 2012), some have theorized that mirror neurons transform perceptual information regarding an intentional action in terms of the observer's own action possibilities (Gazzola et al., 2007). The idea that the hMNS could link perceived actions with appropriate motor plans was confirmed by an fMRI study designed by Ocampo et al. (2011) who studied the neural activations underlying execution of actions that were unlike the ones observed. As expected, activity within the right IPL and right IFG-core regions of the hMNS-was greatest in the imitative context when the participants responded with actions that were similar to the hand actions observed. Interestingly, activity within these regions also increased when dissimilar actions were performed, indicating that there are increased demands linked to remapping stimulusresponse associations (Figure 1A). Shibata et al. (2011) likewise found that the right IFG was involved in mediating higher-order action understanding linked to a complementary action request. Overall, these findings seem to suggest that there are two separate processes and that both are supported by fronto-parietal brain regions. The first process operates at a simple motor level within contexts that require similar responses. The second allows the observer to inhibit those responses and to prepare an action that is compatible with the task demands at hand.

A more integrated description of neural circuits underlying complementary actions was recently outlined by Kokal et al. (2009; Kokal and Keysers, 2010; Figure 1B). Participants in an interactive fMRI study were instructed to carry out complementary and imitative actions in real-time cooperation with an experimenter ("Joint Action"), by performing the same actions individually ("Execution"), or by simply observing the experimenter's actions ("Observation"). This experiment raised our understanding of social interactions to an entirely new level by specifically mapping the contribution of the hMNS (i.e., common voxels for both execution and observation) as well as the areas specifically involved in the joint actions (i.e., voxels exceeding the sum of execution and observation). The areas responsible for this integration process were located bilaterally in the IFG, IPL, precentral gyrus, superior parietal lobule, middle and temporal occipital gyri, and cerebellum.

Two anatomically separate networks have thus been delineated: one that would decipher observed and executed actions into a single common code (Etzel et al., 2008) and another that would integrate this information to successfully achieve common goals. These findings show that although the hMNS plays a critical role in translating all actions into a common code, their flexible remapping seems to be performed elsewhere. It would seem then that any potential discrepancy between an observed action and a complementary response can be resolved flexibly in a two-step manner. During the first step, the observed action is processed in order to predict its goal. During the second, associations are made between the action observed and the appropriate response needed to accomplish a complementary goal. Crucially, Erlhagen et al. (2006) proposed an anatomical model based on animal studies differentiating direct (automatic) and flexible routes for action-perception coupling. The model involves four interconnected brain areas, namely the superior temporal sulcus (STS), area PF (Brodmann area 7b), area F5, and the prefrontal cortex (PFC). The STS-F5 connection, allowing for the matching between a visual description of an action and its motor representation, would represent the neural basis of the direct route for the automatic imitation of an observed action. More importantly, when required, the flexible action-perception coupling is realized in the model by the connection between the PF area and the PFC through which goal representations from the PFC can modulate and set the coupling between visual (STS) and motor (F5) representations (Erlhagen et al., 2006).

Notably, the temporal course of the low- and high-level systems interaction has long been debated.

If output from control systems guide and modulates the mirror system, this would represent a top-down process. The STORM model (social top-down response modulation) suggests that the decision to imitate or to inhibit imitation initially draws on social signals and is most likely supported by a brain network including medial Prefrontal Cortex (mPFC) and temporoparietal junction (TPJ), two core areas of the so-called Mentalizing system, engaged when participants judge other people's mental state (Wang and Hamilton, 2012; Hamilton, 2015). Recently, Cross et al. (2013) have proposed a model for conflict processing in case of incongruence between observed and executed actions. When preparation to avoid imitation is not possible, medial prefrontal regions (mPFC and anterior cingulate cortex) would first detect imitative conflict and send information to anterior insula which would process conflict resolution, suppressing the unwanted motor activation. The hMNS would be therefore the target of top-down mechanisms of conflict resolution. In contrast, if the hMNS leads to an automatic tendency to imitate an observed action and this information feeds up to a monitoring system, this represents a bottom-up process (Brass et al., 2009). According to Ubaldi et al. (2015; see also Barchiesi and Cattaneo, 2013), early mirror responses (150 ms from onset of visual stimuli) would 
be followed by later rule-based non-mirror responses (300 ms). These data seem to indicate that a fast bottom-up process mediated by the dorsal visual stream produces automatic imitative responses. Whereas rule-based visuomotor associations would be mediated by a slower top-down system, relying on the PFC.

\section{Neurophysiologic Studies of Complementary Actions}

Action observation automatically activates corresponding motor representations in an observer, and the stronger support for this process comes from single-pulse transcranial magnetic stimulation (spTMS) over the primary motor cortex (M1) and concomitant electromyography (EMG; e.g., Fadiga et al., 1995). This technique allows to measure modulations in an observer's corticospinal (CS) excitability while he/she watches an agent performing an action. A statistically significant increase in TMS-induced motor evoked potentials (MEP) amplitudes in the corresponding muscles indicates that observers are specifically attuned to the observed action and at what time it does occur. The facilitation of CS excitability provided the first physiological evidence for a direct matching in humans between action perception and action execution (for review, see Fadiga et al., 2005), and made it possible to explore motor system reactions in interactive contexts. A series of recent neurophysiologic studies were designed to assess the facilitation of CS excitability while participants observed videoclips evoking imitative and complementary gestures (Sartori et al., 2011b, 2012, 2013a,b,c). In one of these studies (Sartori et al., 2012), TMS-induced MEPs were recorded from the participants' hand muscles while they observed an actor grasping an object and then unsuccessfully attempting to complete a task (e.g., pouring coffee in a cup which was strategically placed out of her reach but in the video foreground, close to the observer's right hand). An almost imperceptible movement of the actor's hand was interpreted as a request to move the out-of-reach cup closer to the actor so that she could complete the action (Figure 2). Notably, the type of grasp the participant observed and the one that was needed to complete the actor's task were mismatched in all of the videos (i.e., a WHG performed by the actor vs. a PG required of the observer, and vice versa). As the participants were instructed to remain motionless throughout the task, the degree to which the motor system was activated provided an index of the CS activity elicited by action preparation. Moreover, as no explicit instructions were imparted to the participants, the experiment uncovered spontaneous tendencies to fulfill an implicit request embedded in a social interaction. This experiment was particularly enlightening in view of the fact that most studies typically ask participants to perform actions that are not associated with any meaningful behavior in real-world settings or utilize paradigms aiming to uncover dispositions formed during the execution of the experimental task itself (e.g., in imitation vs. complementary blocks) rather than spontaneous tendencies. Study results showed that a matching mechanism at the beginning of an action sequence turned into a complementary one as soon as the request for a reciprocal action became evident (functional shift). The musclespecificity of MEP recordings highlighted the interplay between the initial tendency to resonate with what was observed and

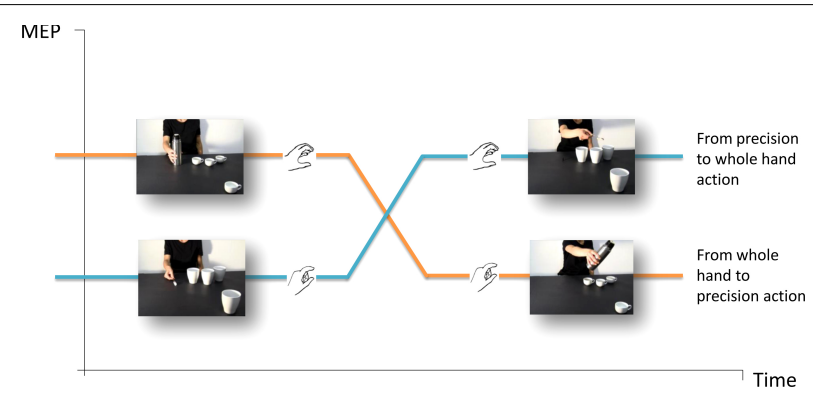

FIGURE 2 | The functional shift. A fundamental requirement for successful complementary actions is the capacity to smoothly and efficiently switch from observing another person's gestures to planning a corresponding reciprocal action. TMS-induced MEPs were recorded from participants' hand muscles in response to observing an actor grasping an object and then trying vainly to fulfill a task (e.g., pouring coffee) in a cup which was strategically placed out of her reach but in the video foreground, close to the observer's right hand (Sartori et al., 2012, 2013b,c). The type of grasp observed and the one that was required were reciprocally mismatched in all the videos (i.e., a WHG performed by the actor vs. a PG requested of the observer, and vice versa).

the subsequent inclination to implicitly prepare for a dissimilar complementary action (Figure 2).

At this point a new important question arose: at what point does this functional switch occur? A new experiment was designed in which TMS was delivered at five different timepoints corresponding to five kinematic landmarks characterizing the observed action (Sartori et al., 2013b,c). The most critical was the fourth $\left(\mathrm{T}_{4}\right)$ timepoint when the actor's hand trajectory began to significantly move toward the out-of-reach object. A TMS pulse was delivered precisely at that moment to investigate whether participants were able to predict the actor's trajectory even before the action became explicit. The control condition that was designed consisted in the actor bringing her hand back to its initial position-with the out-of-reach object still visible in the foreground. The results showed that the participants quickly discriminated between an action driven by a social goal and one that was not, simply by observing the kinematic cues signaling the direction of the actor's hand. These findings have direct implications with regard to action representation theories because they suggest that intention attribution (i.e., social vs. individual) is sensitive to kinematic constraints. As different types of intentional actions have distinctive motion signatures, observers appear to take note of precocious differences in kinematics during action observation in order to be able to predict the actor's intentions (Kilner et al., 2007; Sartori et al., 2009, 2011a; Becchio et al., 2010, 2012a,b; Manera et al., 2011).

\section{A Working Memory Hypothesis}

A dual process seems then to underlie joint actions: a low-level motor resonance analyzes and stores information on observed actions, while a high-level system would flexibly integrate our and others' motor intentions and select the most appropriate response and time course to achieve joint goals (van Schie et al., 2008a). It can be hypothesized then that the hMNS' function is similar to that of a working memory, although specifically tailored for 
action. Mirroring the responses of others might be useful to constantly track and monitor the interacting partners, and to support temporal coordination and action planning (Colling et al., 2013) while cognitive control systems come into play to distinguish selfand other-related representation, to inhibit unwanted imitative responses and to enforce self-generated actions (Brass et al., 2009; Cross et al., 2013). As in the case of the working memory, distinct elements would be kept on-line while others are being processed (Gibson, 2000). We therefore suggest an extension of the previous models of imitation control involving a cross-talk and a simultaneous activation of low- and high-level systems.

Complementary actions are the ideal way to test this topic. During complex social interaction/s, the individual needs to keep information relative to the observed action available while contemporaneously attempting to process a response. In this type of context, the mirror system may be involved in keeping action-related information on hold to enable other brain areas to extract the meaning of the action observed to achieve a joint goal. Notably, observing another person's actions priming for an incongruent reaction can lead to a motor resonant response in the observer's corresponding muscles as well as a simultaneous preparation in different effectors necessary for achieving a complementary response (Sartori et al., 2015). The relation between observed and executed actions seems then to be coordinated by a social associative memory which apparently matches some actions to their natural social responses regardless of who is actually performing the action (Chinellato et al., 2013). Under this model, there would be no difference between congruent, incongruent and complementary responses, as long as they have been associatively linked. In this vein, Catmur et al., (2007; 2008; 2009; see also Heyes, 2001, 2011; Cooper et al., 2013) have proposed that flexibility in action perception

\section{References}

Barchiesi, G., and Cattaneo, L. (2013). Early and late motor responses to action observation. Soc. Cogn. Affect. Neurosci. 8, 711-719. doi: 10.1093/scan/nss049

Becchio, C., Manera, V., Sartori, L., Cavallo, A., and Castiello, U. (2012a). Grasping intentions: from thought experiments to empirical evidence. Front. Hum. Neurosci. 6:117. doi: 10.3389/fnhum.2012.00117

Becchio, C., Cavallo, A., Begliomini, C., Sartori, L., Feltrin, G., and Castiello, U. (2012b). Social grasping: from mirroring to mentalizing. Neuroimage 61, 240-248. doi: 10.1016/j.neuroimage.2012.03.013

Becchio, C., Sartori, L., and Castiello, U. (2010). Toward you: the social side of actions. Curr. Dir. Psychol. Sci. 19, 183-188. doi: 10.1177/0963721410370131

Brass, M., Derrfuss, J., and Von Cramon, D. Y. (2005). The inhibition of imitative and overlearned responses: a functional double dissociation. Neuropsychologia 43, 89-98. doi: 10.1016/j.neuropsychologia.2004.06.018

Brass, M., Ruby, P., and Spengler, S. (2009). Inhibition of imitative behaviour and social cognition. Philos. Trans. R. Soc. Lond. B Biol. Sci. 364, 2359-2367. doi: 10.1098/rstb.2009.0066

Catmur, C., Gillmeister, H., Bird, G., Liepelt, R., Brass, M., and Heyes, C. M. (2008). Through the looking glass: counter-mirror activation following incompatible sensorimotor learning. Eur. J. Neurosci. 28, 1208-1215. doi: 10.1111/j.14609568.2008.06419.x

Catmur, C., Walsh, V., and Heyes, C. (2009). Associative sequence learning: the role of experience in the development of imitation and the mirror system. Philos. Trans. R. Soc. Lond. B Biol. Sci. 364, 2369-2380. doi: 10.1098/rstb.2009.0048

Catmur, C., Walsh, V., and Heyes, C. M. (2007). Sensorimotor learning configures the human mirror system. Curr. Biol. 17, 1527-1531. doi: 10.1016/ j.cub.2007.08.006 coupling may be gained thanks to associative sequence learning (i.e., the ASL theory) developed during social interactions. They strongly suggests that overlearned responses are able to modulate the motor priming effect: when a specific behavior is contingent on a non-matching behavior (e.g., extending the right hand when observing a right hand), an incongruent association is formed.

\section{Conclusion}

The research outlined here shows that motor resonance elicited by action observation is modulated depending on its context: when an observed gesture is socially relevant (i.e., there is an implicit or explicit request) anticipatory complementary activations follow. The assumption that observing an action automatically triggers the inclination to imitate probably arose because most studies did not explicitly challenge the automaticity or flexibility of the visuomotor transformation process. The data outlined here have contributed to shedding light on the functioning of the human motor system in social contexts and on the types of social behavior frequently occurring in real-world settings. From a wider perspective, we can theorize that defining the conditions and the modalities by which motor resonant responses to action observation can be modulated may prove to have specific translational implications leading to the development of novel neuro-rehabilitation protocols for patients with localized lesions to cortical motor areas (e.g., ischemic stroke) and for pathologies such as autism (Hamilton, 2015). More distant horizons may include developing models of brain mechanisms underlying social interactions in the effort to endow artificial agents such as robots with the ability to perform meaningful complementary actions in response to observed actions.

Chinellato, E., Ognibene, D., Sartori, L., and Demiris, Y. (2013). “Time to change: deciding when to switch action plans during a social interaction," in Biomimetic and Biohybrid Systems, eds N. F. Lepora, A. Mura, H. G. Krapp, P. F. M. J. Verschure, and T. J. Prescott (Berlin: Springer), 47-58.

Colling, L. J., Knoblich, G., and Sebanz, N. (2013). How does “mirroring” support joint action. Cortex 49, 2964-2965. doi: 10.1016/j.cortex.2013.06.006

Cooper, R. P., Cook, R., Dickinson, A., and Heyes, C. M. (2013). Associative (not Hebbian) learning and the mirror neuron system. Neurosci. Lett. 540, 28-36. doi: 10.1016/j.neulet.2012.10.002

Costantini, M., Committeri, G., and Sinigaglia, C. (2011). Ready both to your and to my hands: mapping the action space of others. PLOS ONE 6:e17923. doi: 10.1371/journal.pone.0017923

Cross, K. A., Torrisi, S., Losin, E. A. R., and Iacoboni, M. (2013). Controlling automatic imitative tendencies: interactions between mirror neuron and cognitive control systems. Neuroimage 83, 493-504. doi: 10.1016/ j.neuroimage.2013.06.060

de Bruin, L., and Gallagher, S. (2012). Embodied simulation, an unproductive explanation: comment on Gallese and Sinigaglia. Trends Cogn. Sci. 16, 98-99. doi: 10.1016/j.tics.2011.12.003

di Pellegrino, G., Fadiga, L., Fogassi, L., Gallese, V., and Rizzolatti, G. (1992). Understanding motor events: a neurophysiological study. Exp. Brain. Res. 91, 176-180. doi: 10.1007/BF00230027

Erlhagen, W., Mukovskiy, A., and Bicho, E. (2006). A dynamic model for action understanding and goal-directed imitation. Brain Res. 1083, 174-188. doi: 10.1016/j.brainres.2006.01.114

Etzel, J. A., Gazzola, V., and Keysers, C. (2008). Testing simulation theory with cross-modal multivariate classification of fMRI data. PLoS ONE 3:e3690. doi: 10.1371/journal.pone.0003690 
Fadiga, L., Craighero, L., and Olivier, E. (2005). Human motor cortex excitability during the perception of others' action. Curr. Opin. Neurobiol. 15, 213-218. doi: 10.1016/j.conb.2005.03.013

Fadiga, L., Fogassi, L., Pavesi, G., and Rizzolatti, G. (1995). Motor facilitation during action observation: a magnetic stimulation study. J. Neurophysiol. 73, 2608-2611.

Fogassi, L., and Gallese, V. (2002). The neural correlates of action understanding in non-human primates. Adv. Conscious. Res. 42, 13-36. doi: 10.1075/aicr.42.03fog

Gallese, V. (2001). The 'shared manifold' hypothesis. From mirror neurons to empathy. J. Conscious. Stud. 8, 33-50.

Gallese, V., and Sinigaglia, C. (2011). What is so special about embodied simulation? Trends. Cogn. Sci. 15, 512-519. doi: 10.1016/j.tics.2011.09.003

Gazzola, V., van der Worp, H., Mulder, T., Wicker, B., Rizzolatti, G., and Keysers, C. (2007). Aplasics born without hands mirror the goal of hand actions with their feet. Curr. Biol. 17, 1235-1240. doi: 10.1016/j.cub.2007.06.045

Gibson, E. (2000). "The dependency locality theory: a distance-based theory of linguistic complexity” in Image, Language, Brain, eds Y. Miyashita, A. Marantz, and W. O'Niel (Cambridge, MA: MIT Press), 95-126.

Graf, M., Schütz-Bosbach, S., and Prinz, W. (2009). Motor Involvement in Action and Object Perception: Similarity and Complementarity. Grounding Sociality: Neurons, Minds, and Culture. New York, NY: Psychology Press.

Hamilton, A. F. de C. (2015). The neurocognitive mechanisms of imitation. Curr. Opin. Behav. Sci. 3, 63-67. doi: 10.1016/j.cobeha.2015.01.011

Heyes, C. (2001). Causes and consequences of imitation. Trends Cogn. Sci. 5, 253-261. doi: 10.1016/S1364-6613(00)01661-2

Heyes, C. (2011). Automatic imitation. Psychol. Bull. 137, 463-83. doi: 10.1037/a0022288

Kilner, J. M., Friston, K. J., and Frith, C. D. (2007). Predictive coding: an account of the mirror neuron system. Cogn. Process. 8, 159-166. doi: 10.1007/s10339-0070170-2

Knoblich, G., Butterfill, S., and Sebanz, N. (2011). "Psychological research on joint action: theory and data," in The Psychology of Learning and Motivation-Advances in Research and Theory, ed. B. Ross (London: Academic Press), 59-101.

Knoblich, G., and Flach, R. (2001). Predicting the effects of actions: interactions of perception and action. Psychol. Sci. 12, 467-472. doi: 10.1111/1467-9280. 00387

Kokal, I., Gazzola, V., and Keysers, C. (2009). Acting together in and beyond the mirror neuron system. Neuroimage 47, 2046-2056. doi: 10.1016/j.neuroimage. 2009.06.010

Kokal, I., and Keysers, C. (2010). Granger causality mapping during joint actions reveals evidence for forward models that could overcome sensory-motor delays. PLoS ONE 5:e13507. doi: 10.1371/journal.pone.0013507

Longo, M. R., Kosobud, A., and Bertenthal, B. I. (2008). Automatic imitation of biomechanically possible and impossible actions: effects of priming movements versus goals. J. Exp. Psychol. Hum. 34, 489-501. doi: 10.1037/0096-1523.34. 2.489

Manera, V., Becchio, C., Cavallo, A., Sartori, L., and Castiello, U. (2011). Cooperation or competition? Discriminating between social intentions by observing prehensile movements. Exp. Brain Res. 211, 547-556. doi: 10.1007/s00221-0112649-4

Newman-Norlund, R. D., Bosga, J., Meulenbroek, R. G., and Bekkering, H. (2008). Anatomical substrates of cooperative joint-action in a continuous motor task: virtual lifting and balancing. Neuroimage 41, 169-177. doi: 10.1016/j.neuroimage.2008.02.026

Newman-Norlund, R. D., Noordzij, M. L., Meulenbroek, R. G., and Bekkering, H. (2007a). Exploring the brain basis of joint action: co-ordination of actions, goals and intentions. Soc. Neurosci. 2, 48-65. doi: 10.1080/17470910701224623

Newman-Norlund, R. D., van Schie, H. T., van Zuijlen, A. M., and Bekkering, H. (2007b). The mirror neuron system is more activated during complementary compared with imitative action. Nat. Neurosci. 10, 817-818. doi: $10.1038 / \mathrm{nn} 1911$

Ocampo, B., and Kritikos, A. (2010). Placing actions in context: motor facilitation following observation of identical and non-identical manual acts. Exp. Brain Res. 201, 743-751. doi: 10.1007/s00221-009-2089-6

Ocampo, B., Kritikos, A., and Cunnington, R. (2011). How frontoparietal brain regions mediate imitative and complementary actions: an fMRI study. PLoS ONE 6:e26945. doi: 10.1371/journal.pone.0026945
Poljac, E., van Schie, H. T., and Bekkering, H. (2009). Understanding the flexibility of action-perception coupling. Psychol. Res. 73, 578-586. doi: 10.1007/s00426009-0238-y

Rizzolatti, G., Cattaneo, L., Fabbri-Destro, M., and Rozzi, S. (2014). Cortical mechanisms underlying the organization of goal-directed actions and mirror neuronbased action understanding. Physiol. Rev. 94, 655-706. doi: 10.1152/physrev.00009.2013

Rizzolatti, G., and Craighero, L. (2004). The mirror-neuron system. Annu. Rev. Neurosci. 27, 169-192. doi: 10.1146/annurev.neuro.27.070203.144230

Sacheli, L. M., Candidi, M., Pavone, E. F., Tidoni, E., and Aglioti, S. M. (2012). And yet they act together: interpersonal perception modulates visuo-motor interference and mutual adjustments during a joint-grasping task. PLoS ONE 7:e50223. doi: 10.1371/journal.pone.0050223

Sacheli, L. M., Tidoni, E., Pavone, E. F., Aglioti, S. M., and Candidi, M. (2013). Kinematics fingerprints of leader and follower role-taking during cooperative joint actions. Exp. Brain Res. 226, 473-486. doi: 10.1007/s00221-013-3459-7

Sartori, L., Becchio, C., Bulgheroni, M., and Castiello, U. (2009). Modulation of the action control system by social intention: unexpected social requests override preplanned action. J. Exp. Psychol. Hum. Percept. Perform. 35, 1490-1500. doi: $10.1037 / \mathrm{a} 0015777$

Sartori, L., Becchio, C., and Castiello, U. (2011a). Cues to intention: the role of movement information. Cognition 119, 242-252. doi: 10.1016/j.cognition. 2011.01.014

Sartori, L., Cavallo, A., Bucchioni, G., and Castiello, U. (2011b). Corticospinal excitability is specifically modulated by the social dimension of observed actions. Exp. Brain Res. 211, 557-568. doi: 10.1007/s00221-011-2650-y

Sartori, L., Betti, S., and Castiello, U. (2013a). When mirroring is not enough: that is, when only a complementary action will do (the trick). Neuroimage 24, 601-604. doi: 10.1097/WNR.0b013e3283630a66

Sartori, L., Betti, S., and Castiello, U. (2013b). Corticospinal excitability modulation during action observation. J. Vis. Exp. 82, e51001. doi: 10.3791/51001

Sartori, L., Bucchioni, G., and Castiello, U. (2013c). When emulation becomes reciprocity. Soc. Cogn. Affect. Neurosci. 8, 662-669. doi: 10.1093/scan/nss044

Sartori, L., Betti, S., Chinellato, E., and Castiello, U. (2015). The multiform motor cortical output: kinematic, predictive and response coding. Cortex doi: 10.1016/j.cortex.2015.01.019. [Epub ahead of print].

Sartori, L., Cavallo, A., Bucchioni, G., and Castiello, U. (2012). From simulation to reciprocity: the case of complementary actions. Soc. Neurosci. 7, 146-158. doi: 10.1080/17470919.2011.586579

Sebanz, N., Bekkering, H., and Knoblich, G. (2006). Joint action: bodies and minds moving together. Trends Cogn. Sci. 10, 70-76. doi: 10.1016/j.tics.2005.12.009

Shibata, H., Inui, T., and Ogawa, K. (2011). Understanding interpersonal action coordination: an fMRI study. Exp. Brain Res. 211, 569-579. doi: 10.1007/s00221011-2648-5

Ubaldi, S., Barchiesi, G., and Cattaneo, L. (2015). Bottom-up and top-down visuomotor responses to action observation. Cereb. Cortex 25, 1032-1041. doi: $10.1093 /$ cercor/bht295

van Schie, H. T., Koelewijn, T., Jensen, O., Oostenveld, R., Maris, E., and Bekkering, H. (2008a). Evidence for fast, low-level motor resonance to action observation: an MEG study. Soc. Neurosci. 3, 213-228. doi: 10.1080/17470910701414364

van Schie, H. T., Van Waterschoot, B. M., and Bekkering, H. (2008b). Understanding action beyond imitation: reversed compatibility effects of action observation in imitation and joint action. J. Exp. Psychol. Hum. Percept. Perform. 34, 1493-1500. doi: $10.1037 / \mathrm{a} 0011750$

Wang, Y., and Hamilton, A. F. D. C. (2012). Social top-down response modulation (STORM): a model of the control of mimicry in social interaction. Front. Hum. Neurosci. 6:153. doi: 10.3389/fnhum.2012.00153

Conflict of Interest Statement: The authors declare that the research was conducted in the absence of any commercial or financial relationships that could be construed as a potential conflict of interest.

Copyright (C) 2015 Sartori and Betti. This is an open-access article distributed under the terms of the Creative Commons Attribution License (CC BY). The use, distribution or reproduction in other forums is permitted, provided the original author(s) or licensor are credited and that the original publication in this journal is cited, in accordance with accepted academic practice. No use, distribution or reproduction is permitted which does not comply with these terms. 